Gut, 1986, 27, 986-989

Techniques

\title{
Balloon dilatation in achalasia: a new dilator
}

\author{
J COX, G K BUCKTON, AND J R BENNETT \\ From Hull Royal Infirmary, Hull
}

SUMMARY A newly available balloon dilator for the treatment of achalasia and its advantages over existing instruments are discussed. Our experience with its use in seven patients is described.

Achalasia is treated by either forceful 'bag' dilatation or surgical cardiomyotomy. The only prospective randomised comparison of these treatments ${ }^{1}$ was a small series. Other comparisons have been retrospective, and although some suggest that surgery has a better outcome,${ }^{2-4}$ there is evidence that pneumatic dilatation in experienced hands gives good results ${ }^{5-7}$ and its notable advantages - speed and the avoidance of surgery - have led to its increasing popularity.

\section{CURRENT DILATORS}

Pneumatic dilators may be cumbersome to use and none is perfect. The introduction of the dilator may pose special problems. The Hurst-Tucker (BrownMcHardy) has a mercury weighted tip which may not pass through a tortuous oesophagus. The steel tip of the Rider-Moeller dilator may traumatise the oesophagus. The best reported results ${ }^{7}$ are with the Sippy dilator but this is elaborate to prepare and insert and requires serial dilatations using a series of balloon sizes.

Dilators mounted on endoscopes are easy to insert ${ }^{8}$ but the operator may still require fluoroscopy to check that the waist of the balloon is correctly sited. With dumb-bell shaped or waisted balloons it is difficult to know whether the cardia is being dilated by the narrow waist or wider ends. Balloons which are not radio-opaque cannot be accurately sited by fluoroscopy. The use of excessive pressure when using a latex balloon may rupture the inner bag, and by direct transmission of full pressure through the net to the unrestricted outer bag cause

Address for correspondence: Dr J R Bennett. Hull Royal Infirmary. Anlaby Road. Hull HU3 2JZ.

Received for publication 13 December 1985 excessive oesophageal dilatation. Lastly, some models are difficult to obtain in Britain.

We now describe our initial experience with a new dilator.

THE INSTRUMENT

The Rigiflex Achalasia Dilator (Figs 1 and 2) is similar in design to the Grunzig angioplasty catheter. The balloon is made of a modified polyethylene (Polytuff $150^{\mathrm{TM}}$ ) and is mounted on a flexible polyethylene catheter. The balloon is inelastic so that when inflated it maintains its size and shape despite high inflation pressures. Increasing the pressure when the balloon is fully inflated makes it harder but not larger, and therefore a net is not necessary to control its diameter. Should the balloon rupture, the design of Polytuff $150^{\mathrm{TM}}$ ensures that it does so through a safe longitudinal tear rather than a damaging transverse 'blow-out'. The burst pressure is about $140 \mathrm{kPa},(20 \mathrm{psi}, 1050 \mathrm{mmHg})$. The $10 \mathrm{~cm}$ long balloon inflates to a maximum diameter of $30 \mathrm{~mm}, 90 \mathrm{Fr}$. The uninflated diameter of $4.7 \mathrm{~mm}$, $14 \mathrm{Fr}$ compares well with the tips of the Mosher ( $8 \mathrm{~mm}, 24 \mathrm{Fr})$, the Hurst-Tucker $(12 \mathrm{~mm}, 36 \mathrm{Fr})$, and the Rider-Moeller $(12.5 \mathrm{~mm}, 38 \mathrm{Fr})$ and the diameters of the undilated Hurst-Tucker and RiderMoeller ( $18 \mathrm{~mm}, 54 \mathrm{Fr})$. The catheter on which the balloon is mounted has a soft, tapered, radioopaque tip made of Percuflex ${ }^{\mathrm{TM}}$ which is said to allow easy placement, but for added safety we have chosen to pass it over a Teflon coated guide wire. Radio-opaque tantalum markers proximal and distal to the balloon help in positioning the uninflated balloon at fluoroscopy. The balloon can be inflated with air or contrast medium - we chose Urografin 150 to provide good visibility. The air should be 


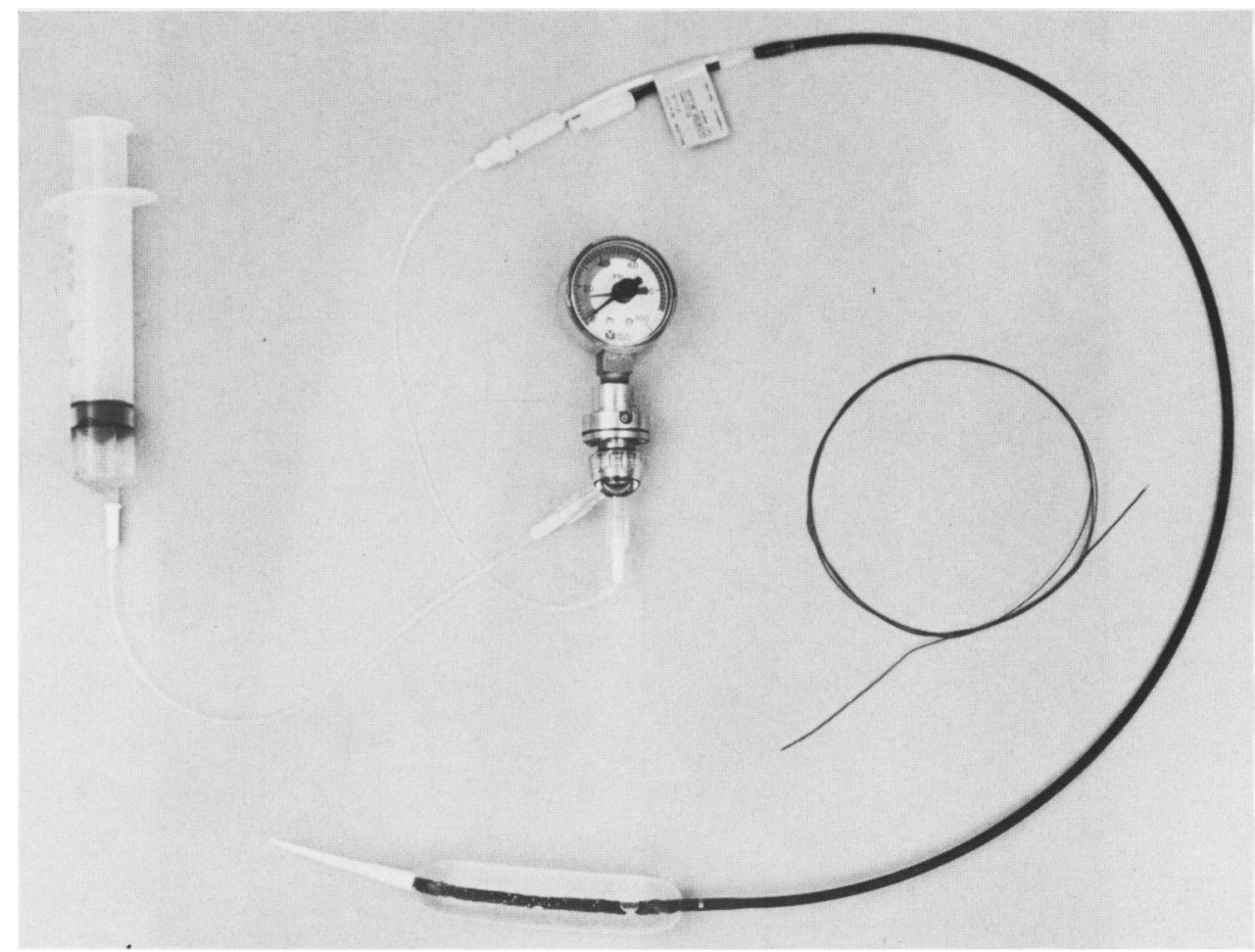

Fig. 1 The Rigiflex achalasia dilator inflated.

leached entirely from the balloon before use, as the incompressible contrast medium then provides accurate and reproducible pressure measurements. The balloon is cleaned in Dettox and cold sterilised in activated gluteraldehyde. If kept cool and dry, the balloon has an unlimited shelf life, unlike the latex balloons. It is manufactured by Rigiflex and distributed in England by KeyMed.

\section{Method}

\section{PATIENTS}

The patient is sedated with intravenous diazepam and an oesophageal washout carried out. The guide wire is inserted through a $3 \mathrm{~mm}$ (yellow Kifa) catheter or through the biopsy channel of a fibreoptic endoscope and the balloon, connected to a pressure gauge and a syringe of contrast medium is passed over the guide wire with fluoroscopic control. After partial inflation of the balloon by contrast medium its position is adjusted until the constriction produced by the tonic gastro-oesophageal sphincter is at the midpoint of the balloon (Fig. 2). Further inflation then produces a cylindrical profile of $30 \mathrm{~mm}, 90 \mathrm{Fr}$ diameter which is maintained for 60 seconds (requiring pressures ranging from 14 to $104 \mathrm{kPa}$ (2-15 psi, 105-780 $\mathrm{mmHg}$ ). The contrast is evacuated by syringe and the balloon removed. The patient is carefully observed overnight and allowed to drink only sterile water until the next morning.

\section{Results}

Forceful dilatation of the cardia using this balloon has been accomplished in seven patients with achalasia. These were three men and four women aged 28-59 years. Achalasia was diagnosed on history, cine-barium studies, intraluminal $\mathrm{pH}$ studies, manometry, and endoscopy. One patient had had two oesophageal dilatations under general anaesthetic, 10 and eight years previously and had subsequently passed a Hurst mercury dilator twice weekly. Another had a Hurst-Tucker dilatation four years previously and a Rider-Moeller dilatation two years previously, and a third had received an incomplete dilatation with the Hurst-Tucker instrument 10 years earlier. The other four patients had not received any treatment previously. The oesophagus 

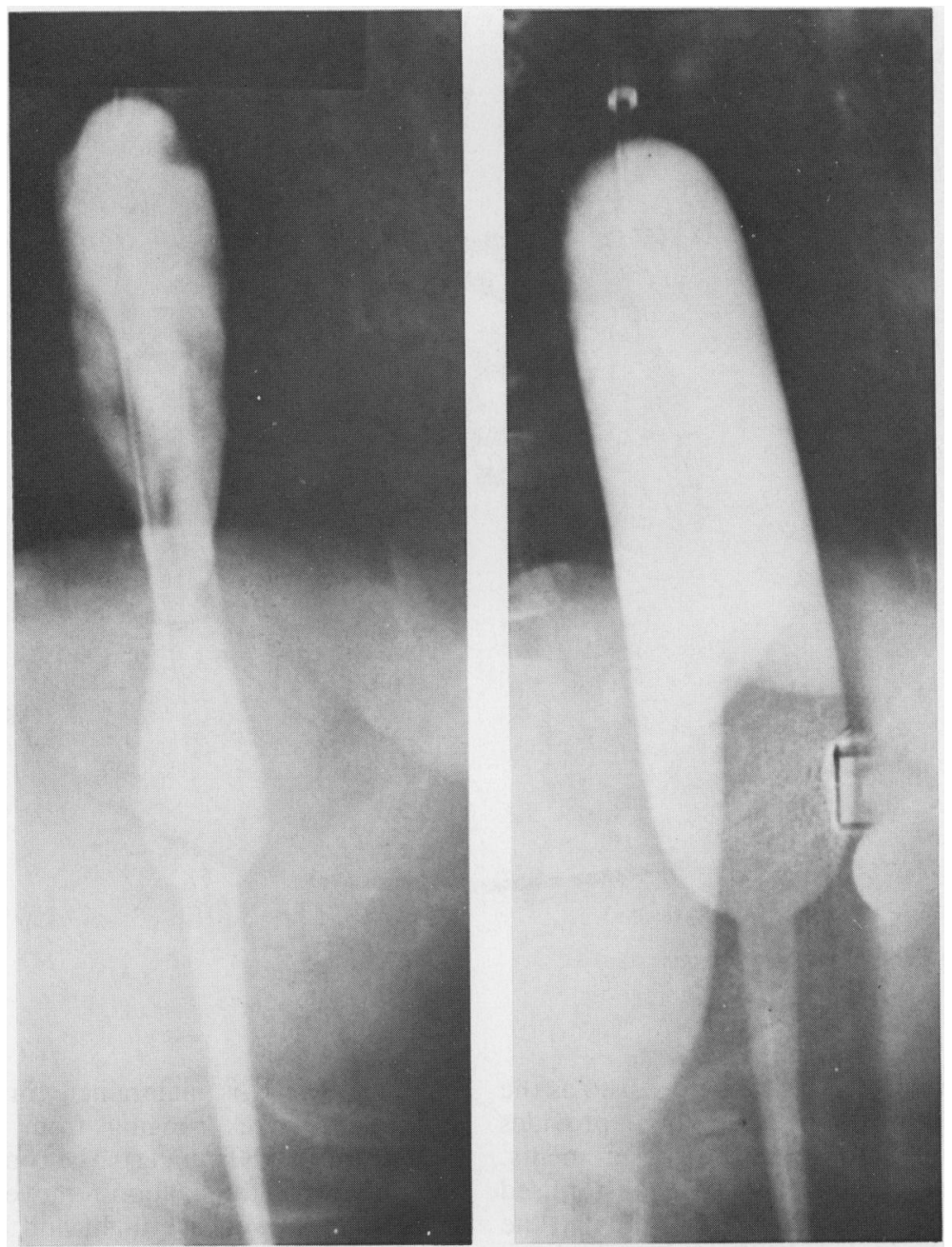

Fig. 2 Radiograph of balloon dilator in use (a) (lefthand print) initial inflation showing 'waist' (b) (righthand print) fully inflated to $30 \mathrm{~mm}(90 \mathrm{Fr})$ diameter.

was markedly dilated in five patients, slightly dilated in two patients and was tortuous in two patients. All the dilatations with the new balloon were uneventful, and there was no significant bleeding and no perforation. The patients left hospital the following day having eaten a good breakfast.

At review four weeks later all patients reported improvement in their symptoms without dysphagia or heartburn and repeat erect radiology showed satisfactory flow of barium through the cardia. The follow up has been short: 6-12 months, (mean 9.6 months). Six of the seven patients remain well. The patient who had a Hurst-Tucker and a RiderMoeller dilatation previously, developed a recur- rence of his symptoms eight months later and manometry revealed the lower oesophageal sphincter pressure was still raised at $4.9 \mathrm{kPa}(50 \mathrm{~cm}$ water). He has now been referred for surgery.

\section{Discussion}

Pneumatic dilatation in good hands is a safe, simple, and effective treatment of achalasia. This balloon increases the margin of safety and ease of dilatation by dispensing with some of the difficulties associated with traditional dilators. It is easy to set up and the guide wire, the flexible tip, and the small diameter of the undilated balloon make it easy to introduce. 
The contrast medium enables the operator to ensure that the gastro-oesophageal sphincter is at the midpoint of the balloon and it is impossible to dilate the sphincter beyond the maximum diameter of the balloon. Should the balloon rupture it does so through a safe longitudinal tear. The dilatation is accomplished at a single sitting under diazepam sedation and general anaesthesia is unnecessary. Should the clinician wish to carry out the dilatation with a series of dilators of increasing size, as in Louvain, ${ }^{7}$ similar balloons are available from $20 \mathrm{~mm}$ to $40 \mathrm{~mm}$ in diameter. The balloon is durable, does not perish, is easily sterilised and is readily available. $^{*}$

${ }^{*}$ The Rigiflex Achalasia Dilator. Price $(30 \mathrm{~mm}$ balloon) $£ 522 \cdot 10$ including VAT, from KeyMed, KeyMed House, Stock Road, Southend-on-Sea, Essex SS2 $5 \mathrm{QH}$.

\section{References}

1 Csendes A, Velasco N, Braghetto I, Henriquez A. A prospective randomised study comparing forceful dilatation and esophagomyotomy in patients with achalasia of the oesophagus. Gastroenterology 1981; 80: 789-95.

2 Yon J, Christensen J. An uncontrolled comparison of treatment for achalasia. Ann Surg 1975; 182: 672-6.

3 Okike N, Payne WS, Neufeld DM et al. Esophagotomy versus forceful dilatation for achalasia of the esophagus: results in 899 patients. Ann Thorac Surg 1979; 28: 119-25.

4 Arvanitakis C. Achalasia of the esophagus. A reappraisal of esophagomyotomy vs forceful pneumatic dilatation. Dig Dis 1975; 20: 841-6.

5 Bennett JR, Hendrix T R. Treatment of achalasia with pneumatic dilatation. In: Bayless TM, ed. Management of esophageal disease modern treatment. Vol. 7, no. 6. New York: Harper and Row, 1970.

6 Fellows IW, Ogilvie AL, Atkinson M. Pneumatic dilatation in achalasia. Gut 1983; 24: 1020-3.

7 Vantrappen G, Hellemans J. Treatment of achalasia and related motor disorders. Gastroenterology 1980; 79: $144-54$.

8 Witzel L. Treatment of achalasia with a pneumatic dilator attached to a gastroscope. Endoscopy 1981; 13: 176-7. 